\title{
Cataclysmic Variables and a Candidate Helium White Dwarf in the Globular Cluster NGC 6397
}

\section{Citation}

Edmonds, Peter D., Jonathan E. Grindlay, Adrienne Cool, Haldan Cohn, Phyllis Lugger, and Charles Bailyn. 1999. "Cataclysmic Variables and a Candidate Helium White Dwarf in the Globular Cluster NGC 6397." The Astrophysical Journal 516 (1): 250-62. https:// doi.org/10.1086/307106.

\section{Permanent link}

http://nrs.harvard.edu/urn-3:HUL.InstRepos:41399827

\section{Terms of Use}

This article was downloaded from Harvard University's DASH repository, and is made available under the terms and conditions applicable to Other Posted Material, as set forth at http:// nrs.harvard.edu/urn-3:HUL.InstRepos:dash.current.terms-of-use\#LAA

\section{Share Your Story}

The Harvard community has made this article openly available.

Please share how this access benefits you. Submit a story.

Accessibility 


\title{
Cataclysmic Variables and a Candidate Helium White Dwarf in the Globular Cluster NGC $6397^{1}$
}

\author{
Peter D. Edmonds \& Jonathan E. Grindlay \\ Harvard College Observatory, 60 Garden Street, Cambridge, MA 02138 \\ Adrienne Cool \\ Department of Physics and Astronomy, San Francisco State University \\ 1600 Holloway Avenue, San Francisco, CA 94132 \\ Haldan Cohn \& Phyllis Lugger \\ Department of Astronomy, Indiana University, Swain West \\ Bloomington, IN, 47405 \\ and \\ Charles Bailyn \\ Department of Astronomy, Yale University, P.O. Box 208101 New Haven, CT, 06520-8101
}

\begin{abstract}
We have used the Hubble Space Telescope $(H S T)$ and the Faint Object Spectrograph (FOS) to study faint UV stars in the core of the nearby globular cluster NGC 6397. We confirm the presence of a 4th cataclysmic variable (CV) in NGC 6397 (hereafter CV 4), and we use the photometry of Cool et al. (1998) to present evidence that CVs 1-4 all have faint disks and probably low accretion rates. By combining these results with new UV spectra of CV 1 and the published spectra of Grindlay et al. (1995) we present new evidence that CVs 1-3 may be DQ Her systems, as originally suggested by Grindlay et al. (1995), and we show that CV 4 may either be a dwarf nova or another magnetic system. Another possibility is that the CVs could be old novae in hibernation between nova eruptions (Shara et al. 1986).

We also present the first spectrum of a member of a new class of UV bright stars in NGC 6397 (Cool et al. 1998). These faint, hot stars do not vary, unlike the CVs, and are thus denoted as "non-flickerers" (NFs). Like the CVs, their spatial concentration is strongly concentrated toward the cluster center. Using detailed comparisons with stellar atmosphere models we have determined $\log \mathrm{g}=6.25 \pm 1.0$, and $T_{\text {eff }}=17,500 \pm$ $5,000 \mathrm{~K}$ for this NF. Using these line parameters and the luminosity of the NF we show
\end{abstract}

\footnotetext{
${ }^{1}$ Based on observations with the NASA/ESA Hubble Space Telescope obtained at the Space Telescope Science Institute, which is operated by Association of Universities for Research in Astronomy, Incorporated, under NASA contract NAS 5-26555
} 
that the NF spectrum is consistent with a helium WD having a mass of $\sim 0.25 M_{\odot}$ and an age between 0.1 and 0.5 Gyr (depending on the models used). The NF spectrum appears to be significantly Doppler shifted from the expected wavelength, suggesting the presence of a dark, massive companion, probably a carbon-oxygen (CO) WD.

\section{Introduction}

Binaries have long been thought to have a crucial impact on globular cluster dynamics and evolution (Hut et al. 1992), but only recently (especially with the use of $H S T$ ) have large numbers of them been found in globular cluster cores where they are expected to act as the central energy source that drives cluster expansion. Discoveries of binary millisecond pulsars (e.g. Manchester et al. 1991) and multiple low-luminosity X-ray sources (Hertz \& Grindlay 1983) have recently been supplemented by discoveries of large numbers of eclipsing binaries in globulars (e.g. 47 Tuc;

Edmonds et al. 1996 and Kaluzny et al. 1998) and a significant population of main sequence-main sequence binaries in NGC 6752 (Rubenstein and Bailyn 1997). Another recent breakthrough has been the discovery of cataclysmic variables $(\mathrm{CVs})$ in the cores of globular clusters, using either dwarf nova (DN) outbursts in M5 (Oosterhoff 1941), 47 Tuc (Paresce \& De Marchi 1994) and NGC 6624 (Shara, Zurek \& Rich 1996), UV excess to recover an old nova in M80 (Shara and Drissen 1995) or narrow-band $\mathrm{H} \alpha$ emission. Using the latter technique $3 \mathrm{CVs}$ have been reported in NGC 6397 by Cool et al. (1995) and Grindlay et al. (1995; hereafter GC95), and a fourth CV candidate by Cool et al. (1998; hereafter CG98). Also, 2 probable CVs have been reported in NGC 6752 by Bailyn et al. (1996). These CVs appear to be the long-sought optical counterparts of the low-luminosity X-ray sources found in globular cluster cores. In particular, the 3 brightest optical emission line objects in NGC 6397 (GC95) are the probable counterparts of the 3 brightest X-ray sources found by Cool et al. (1993; see also Cool et al. 1995).

Observations of CVs in clusters can be used for a variety of studies including: (1) CV formation and evolution in low-metallicity environments, (2) stellar interactions in high-density environments, and (3) cluster dynamical evolution. Probable formation mechanisms for globular cluster CVs include tidal capture and exchange collisions between main sequence (MS) stars and white dwarfs (WDs), complementing studies of the MS star - MS star interactions that produce blue stragglers. Since these formation mechanisms differ from those for field CVs, and the stellar environment is different, it would not be surprising to find systematic differences between globular cluster and field CVs. In particular, GC95 and Grindlay (1996) have suggested that the CVs in NGC 6397 might have a much higher percentage of magnetic WDs than field CVs. The dense, collapsed core of NGC 6397 is a prime region to study the effects of stellar interactions because its high central density makes interaction rates large and its relative proximity at $2.2 \mathrm{kpc}$ makes it possible to probe the core with high spatial resolution (CG98).

This paper presents new HST/FOS spectra of CV 1 (from GC95) and CV 4 (from CG98) in NGC 6397. By combining all available spectra for CVs 1-4 with the photometry of CG98 and 
comparing with field CVs we show that the CV disks are consistent with those of faint quiescent DNe, given their expected periods, but that their He II $\lambda 4686$ lines are unusually strong for DNe (such systems would probably have long recurrence times between outbursts). Instead, we argue that CVs 1-3 may be magnetic CVs (or perhaps old novae), and that CV 4 is either a low accretion rate $\mathrm{DN}$ or a magnetic $\mathrm{CV}$. It is possible that these objects may even be quiescent LMXBs, although this is unlikely based on detailed comparisons with the x-ray and optical properties (Grindlay 1996, 1998). In any case we present good evidence that there are systematic differences between populations of globular cluster and field CVs.

Along with the 3 previously known classes of UV bright stars in NGC 6397 (blue stragglers, CVs and WDs), CG98 have discovered another class of UV bright stars. Three faint, hot stars have been found within only $\sim 16^{\prime \prime}$ of the cluster center, all of them non-variable (unlike the flickering CVs). CG98 have argued that these non-flickering (NF) stars are unlikely to be CVs, "normal" CO WDs (recently evolved from single red giants), extended horizontal branch stars or field stars, but instead that they are good candidates for low-mass helium WDs.

Helium WDs have masses $\lesssim 0.49 M_{\odot}$, and in the field are usually found in binary systems containing either another WD or a neutron star (Marsh, Dhillon \& Duck 1995, and Rappaport et al. 1995). These double degenerates are thought to form by Roche lobe overflow (and usually common envelope events) in primordial binaries containing red giants, if He ignition in the red giant core, a proto helium WD, is avoided (Iben, Tutukov and Yungelson 1997 discuss detailed formation scenarios). Several low-mass WDs have been found or inferred in open and globular clusters including the helium WD - red giant binary S1040 in M67 (Landsman et al. 1997), and the ultra-short period X-ray binary systems 4U 1820-30 in NGC 6624 (Anderson et al. 1997) and Star S in NGC 6712 (Anderson et al. 1993). S1040 in M67 probably formed after a subgiant underwent Roche lobe overflow in a primordial binary (Landsman et al. 1997), but in denser globular clusters, primordial binary evolution may be less important than interactions involving subgiants or red giants. For example, red giant/WD or red giant/MS star direct collisions should cause a helium WD to be left behind in a binary system (Davies, Benz \& Hills 1991). Systems such as $4 \mathrm{U}$ 1820-30 and Star S probably result from either neutron star/red giant collisions (Verbunt 1987) or neutron star/MS star capture and delayed mass transfer (Bailyn \& Grindlay 1987).

Here, we report the first spectrum of one of the NGC 6397 NFs. The lack of emission lines in the spectrum provides extra evidence against the CV possibility and the log g value presented here argues against a CO WD or extreme horizontal branch identification. By comparing with published model atmospheres we determine log g and $T_{\text {eff }}$ for the NF and we then compare these parameters (along with the luminosity) with WD evolutionary models to show that a low-mass helium WD is, indeed, a plausible explanation for the NF. We also present evidence that the NF spectrum is significantly Doppler shifted from the expected wavelength, suggesting that the NF is in a binary system with a massive dark companion. 


\section{Observations and Analysis}

\subsection{1}

Spectroscopic observations with $H S T$ were made of CV 1, the brightest CV of the 3 studied by GC95, on October 2nd, 1996. Ultraviolet and optical observations were obtained with the

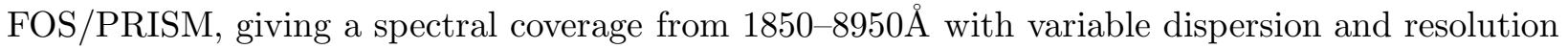
decreasing from the blue to the red. Ultraviolet observations were obtained with the FOS/G160L grating, with a spectral coverage from $1150-2510 \AA$ (at a spectral resolution of $9.2 \AA$ ), including a small contribution from second-order geocoronal Ly $\alpha$ near the red end. The data were originally reduced using the normal pipeline processing but were then recalibrated using updated flat-fields and inverse sensitivity files (removing some flat-field features at around the $5 \%$ level).

Figure 1 summarizes all of the FOS data available for CV 1. The full G160L spectrum is shown and then the PRISM spectrum from $2510 \AA$ red-ward, along with the red G570H spectrum (with a resolution of $4.5 \AA$ ) from Cycle 4. No scaling was used for either the G160L or PRISM spectra, showing good self-consistency in the spectral calibration and the photometric states of (variable) CV 1 between the two separate observations. Despite the low spectral resolution of the PRISM in the blue, the $\mathrm{H} \gamma$ and $\mathrm{H} \delta$ emission lines are visible, along with some absorption lines from the secondary (for example MgII $\lambda 2800$ and the Ca H/K doublet). Since NGC 6397 lies close to the galactic plane its reddening is significant and therefore Figure 1 also shows a dereddened version of the smoothed PRISM spectrum (using $E(B-V)=0.17$ from Alcaino et al. 1997).

Using the WFPC2 photometry from CG98 we estimated the UV and optical contribution from the secondary component in CV 1 . We began by noting that CV 1 is almost on the MS in the $V$ vs $V-I$ CMD, which implies a bright secondary and relatively faint disk for this system. Then, assuming that all of the flux in $I$ comes from the secondary we used the position of the MS in CG98 to estimate $B$ and $V$ for the secondary (showing the advantage of observing binaries in a cluster). We then chose the reddened Kurucz atmosphere $(\log Z=-2.0)$ which best matched the $B V I$ photometry for the secondary. Figure 1 shows the Kurucz stellar atmosphere with a dotted line.

A close-up of the G160L spectrum is shown in Figure 2. The dashed line shows a reddened blackbody fit to the UV spectrum (temperature $=12850 \mathrm{~K}$, without removing the small contribution of the secondary) and the solid line shows dereddening of this fit using $E(B-V)=0.17$. The detection of Ly $\alpha$ in second order and a marginal $(3.5 \sigma)$ detection of He II $\lambda 1640$ are labeled. No other UV lines are detected, and we set $3 \sigma$ upper limits on the equivalent

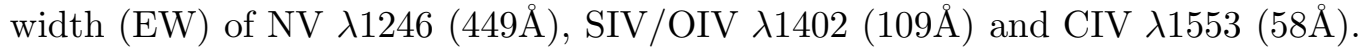

\footnotetext{
${ }^{2}$ Note that: (1) we use the term "disk" loosely for any hot, accretion related element of the binary system, and (2) in general the total CV light minus the secondary will have contributions from both the disk and WD, however the WD component is likely to be negligible, particularly in the $B$ and $V$ passbands.
} 
The most striking feature of the G160L and PRISM spectra is the relatively low UV flux. We have compared the measured flux distribution of CV 1 with that of various subclasses of field CVs studied by Verbunt (1987) with IUE. This comparison has limitations, as pointed out by the referee, since Verbunt's sample may not include the full range of CV properties (including highly magnetic WD primaries and a possible lack of disks in some DQ Hers). However, the data set and analysis is homogeneous and covers most CV classes, with the notable exception of AM Her systems (see below).

Verbunt (1987) determined the fluxes of CVs in $80 \AA$ width bins centered on 1460, 1800, 2140 and $2880 \AA$. We first normalized each (quiescent) system in Verbunt's study to have the same flux at $1460 \AA$ and then averaged the fluxes at other wavelengths over each class. We then predicted, for each CV class, the average UV fluxes expected for $V=18.27$, the magnitude of CV 1 ( $V$ magnitudes are also given in Verbunt 1987). We found reddened fluxes of $F_{1460}=4.3 \times 10^{-16} \mathrm{ergs}$ $\mathrm{cm}^{-2} \mathrm{~s}^{-1} \AA^{-1}$ (DQ Hers), $4.0 \times 10^{-16}$ (U Gems) and $4.5 \times 10^{-16}$ (Z Cams), and even brighter for other CV classes (for example $F_{1460}=1.2 \times 10^{-15}$ for old novae). These fluxes are all over ten times brighter than the observed (reddened) $F_{1460}$ for CV 1 (see Figures 1 and 2). However, scaling by the $V$ magnitude of the system has limited usefulness because the secondary is more massive and brighter than most field CVs.

A better comparison is to calculate relative fluxes in the UV for CV 1, where the contribution from the secondary is less important. The dereddened UV fluxes for CV 1 are shown in Figure 3 along with fluxes for various CV classes (the error bars combine uncertainties in the continuum level estimation and the reddening). Not surprisingly, the reddest flux distributions are found for quiescent dwarf novae (DNe), characterized by low accretion rates, and DQ Her systems, with inner disks truncated by the magnetic field of the WD. Clearly, CV 1 has a much redder UV flux distribution than the field CVs in Verbunt's sample. Figure 3 also plots the flux distribution for the CV 1 disk after subtracting off the secondary and shows that the disk is marginally redder, given the large errors, than all classes of field CV (without the secondaries subtracted).

\section{2. $\quad$ CV 4}

We have obtained an optical spectrum of a fourth CV candidate discovered near the center of NGC 6397 (CG98). Figure 4 shows the G570H spectrum of this star, after correcting for diffuse light (from a combination of extended PSF halos from bright stars such as giants and diffuse light from faint cluster stars). For comparison, we also show an average of the spectra of CVs 1-3 from GC95, where the continua of CVs 2 and 3 were normalized to that of CV 1. Strong Balmer emission lines are present in the spectrum of the new CV candidate along with several HeI lines

and He II $\lambda 4686$. The spectrum confirms that this star is a CV, as suggested by its UV excess and flickering (CG98). The relative weakness of the He II line is the most obvious difference between CV 4 and the average spectrum of CVs 1-3 (see a quantitative analysis below). The spectrum of $\mathrm{CV} 4$ has the highest signal to noise $(\mathrm{S} / \mathrm{N})$ ratio of the $4 \mathrm{CV}$ spectra, enabling easy detection of 
relatively weak lines such as HeI $\lambda 4713$. The dotted line shows the estimated contribution of the secondary, using the procedure outlined above for CV 1.

To improve the spectra, we applied boxcar smoothing over 7 channels (this improved the S/N ratio per independent channel by a factor of 2.6 at $\mathrm{H} \beta$, but in simulations had a negligible effect on measured line parameters). We found that a Voigt profile gave optimal fits to the emission lines, after also experimenting with Gaussians and Lorentzians.

Before deriving EWs (see Section 2.3) and integrated magnitudes from the spectrum of this faint $\operatorname{star}(V=20.81)$, we considered 2 possible additional sources of light in the $0.43^{\prime \prime}$ aperture used: (1) possible light from individual neighboring stars and (2) the diffuse light contribution. By examining the high S/N WFPC2 images of CG98 we found that light from neighbors is negligible, since CV 4 has no measurable companions $<0.93^{\prime \prime}$ away, but that diffuse light is an important factor for this star. To measure the spectral contribution of the diffuse light we used Kurucz (1993) stellar atmospheres to fit a single temperature model to the diffuse light components in $B, V$ and $I$ derived by CG98. A $6,000 \mathrm{~K} \mathrm{Kurucz}$ model gave an excellent fit and showed that relatively hot stars dominated the diffuse light at the position of CV 4 . Around $45 \%$ of the flux at $5500 \AA$ comes from the diffuse light showing the importance of the above correction for accurate EWs. By comparing the CV 4 spectrum with the Kurucz spectrum of the secondary (see Figure 4) we see some evidence for a small residual red component. This component is too red to be caused by the CV disk (or WD), and is perhaps caused by earthshine (a red component also appears, for data near the earth limb, to be present in the spectrum of the NF; see below).

\subsection{He II $\lambda 4686$ line strength}

An important diagnostic for CVs are the line fluxes, especially the relative strength of He II $\lambda 4686$. Table 1 gives values of the wavelength, line flux, EW and FWHM (Gaussian and Lorentzian) of the $\mathrm{H} \beta, \mathrm{H} \alpha$, HeI $\lambda 5876$ and He II $\lambda 4686$ emission lines for CVs $1-4$ ( $1 \sigma$ errors are quoted). We have not undertaken a complete analysis for CVs $1-3$, which requires taking account of light contaminants in the FOS aperture (CV 2, for example, has considerable contamination from a bright neighboring star). However, the line ratio between He II $\lambda 4686$ and $\mathrm{H} \beta$ will be much less sensitive to contamination errors than the EWs and so we derive these ratios from the uncorrected spectra, after taking into account blends from HeI $\lambda 4713$. Since multiline fitting in IRAF 3 /SPLOT was unable to separate the heavily blended He II $\lambda 4686$ and HeI $\lambda 4713$ lines in CVs $1-3$, we used the relative strength of HeI $\lambda 4713$ compared to HeI $\lambda 5876$ for CV 4 (where the blend from the weak He II $\lambda 4686$ line was small; see Figure @) to remove the HeI $\lambda 4713$ line from these systems.

\footnotetext{
${ }^{3}$ IRAF is distributed by the National Optical Astronomy Observatories, which are operated by the Association of Universities for Research in Astronomy, Inc., under cooperative agreement with the National Science Foundation.
} 
The He II $\lambda 4686 / \mathrm{H} \beta$ line ratio for $\mathrm{CV} 4$ is only $0.07 \pm 0.01$, compared to $0.32 \pm 0.04,0.34 \pm$ 0.05 and $0.25 \pm 0.03$ for CVs $1-3$ respectively (these more accurate determination replace those given in GC95). Two other notable differences are that the lines of CV 4 are narrower (smaller FWHMs) than those of CVs 1-3 and the EWs of most of the CV 4 lines are greater than those of CVs 1-3. This latter result is part of a very clear trend that the EWs increase going from CV 1 to CV 4, mainly because the secondaries (which dominate the optical flux) get fainter, without significant changes in the line fluxes. For example, comparing CV 4 with CV 1, the ratio of EWs of $\mathrm{H} \beta$ is 8.8, with a factor of 0.08 difference in the continuum levels and only a factor of 0.7 difference in line fluxes.

To compare the measured He II $\lambda 4686 / \mathrm{H} \beta$ line ratios with those of field CVs we used the emission line data of Williams (1983) and Echevarria (1988) for various CV classes (after confirming the CV classification using Ritter \& Kolb 1998). Because He II $\lambda 4686$ is often quite a weak line and it is highly variable only a subset of the CVs in Echevarria's sample include He II $\lambda 4686$, but even with this relatively small sample, some clear trends emerge. The average He II $\lambda 4686 / \mathrm{H} \beta$ line ratios for the non-magnetic CV classes are $0.16 \pm 0.05(17 \mathrm{DNe}), 0.94 \pm 0.50$ (8 old novae) and $0.76 \pm 0.51$ (13 nova-likes, excluding magnetic systems). These values are probably overestimates (although we lack information about upper limits), especially for DNe where only 17 out of 39 systems in the above sample have measurable He II $\lambda 4686$ (the completeness for the other systems is $8 / 10$ for old novae and 13/17 for nova-likes). For magnetic systems we used the classifications by Patterson (1994) and Ritter \& Kolb (1998) and the spectra of Williams (1983) to calculate an average He II $\lambda 4686 / \mathrm{H} \beta$ line ratio of $0.57 \pm 0.46$ (measurable He II $\lambda 4686$ for $7 / 8$ DQ Her systems) and $0.59 \pm 0.21$ (4/4 AM Her systems). The high accretion rate novae and nova-likes can have relatively strong He II $\lambda 4686$ (probably because of very hot inner disks), while the magnetic systems also usually have strong He II $\lambda 4686$, thought to be because the hot accretion "curtains" along the magnetic field lines of the WD are directly visible. Only the lower accretion rate DNe have relatively weak He II $\lambda 4686$ compared to $\mathrm{H} \beta$.

\subsection{Disk brightness}

For comparison with field CVs we have also estimated the brightness of the disk in CVs 2-4, using the techniques described in Section 2.1 to subtract off the secondary. Table 2 shows the total absolute magnitudes of CVs 1-4, plus estimated absolute magnitudes and masses $\left(M_{2}\right)$ of the secondaries (from Bergbusch and Vandenberg 1992), and absolute magnitudes of the disks $\left(M_{V}\right.$ (disk)). Since Smith and Dhillon (1998) have shown that secondaries in CVs with orbital periods below $\sim 7 \mathrm{~h}$, as likely here (see discussion below) are typically not evolved, the mass estimates for main sequence stars should be reasonable. Note that, using this technique, we found that the $V$ magnitude of the secondary in $\mathrm{CV} 1$ was 0.02 mag brighter than the $V$ magnitude of the total system, clearly an impossibility. Variability is the likely explanation, plus errors will also play a part; to investigate the sensitivity of these results to overestimating the brightness of the 
secondary (and other errors) we made the estimated $I$ magnitude of the secondary fainter by 0.1 mag and $0.2 \mathrm{mag}$ and rederived the expected $M_{V}$ (disk) (see Table 2).

We also show in Table 2, estimates of upper limits for periods of CVs 1-4, using the relationship given in Warner (1995) between the mass of a Roche-lobe filling secondary and the

CV period, namely $M_{2}=0.065 P_{\text {orb }}^{5 / 4}(\mathrm{~h})$. Since this equation applies to Pop I stars and Pop II stars have smaller radii for a given mass than Pop I stars (implying a smaller period for Roche-lobe overflow), we have used the study by Stehle, Kolb \& Ritter (1997) to estimate the period correction required for Pop II stars $\left(<1 \mathrm{~h}\right.$ for all masses $\left.<0.9 M_{\odot}\right)$. As a guide to the validity of this procedure for PopII stars we note that the two CV candidates in NGC 6752 (Bailyn et al. 1996), have absolute $V$ and $I$ magnitudes only a few tenths of a magnitude different from CVs 2 and 3 in NGC 6397. The estimated periods of $4.4 \mathrm{~h}$ and $3.8 \mathrm{~h}$ for CVs 2 and 3 compare well with the observed periods of $5.1 \mathrm{~h}$ and $3.7 \mathrm{~h}$ for the NGC $6752 \mathrm{CV}$ candidates.

\subsection{Non Flickerer}

The G570H spectrum of the NF is shown in Figure 5. The blue continuum and broad $\mathrm{H} \beta$ line suggest that this star is a high gravity object (see below). The spectrum suffers from both diffuse light contamination and from having a much brighter $(\Delta \mathrm{V}=3.5 \mathrm{mag})$ neighbor only $0.3^{\prime \prime}$ distant (a star near the MS turnoff). With perfect pointing during the FOS observations we would simply be able to use the high S/N WFPC2 images from CG98 to determine the exact amount of turnoff star contamination. However, there are $0.08^{\prime \prime}$ uncertainties in the pointing of HST which can potentially make a significant difference to the amount of light contamination. There is also a source of variable light, since the apparent flux of the star increases by about $15 \%$ towards the end of an orbit (probably due to increased scattered light or earthshine as noted above).

Since it is difficult to estimate the "clean", uncontaminated spectrum of this star we forced its continuum to equal that of a reddened Kurucz atmosphere constrained by the $B, V$ and $I$ measurements of CG98. A $\log \mathrm{Z}=-2.0$ Kurucz spectrum was normalized so that the equivalent $V$ magnitude agreed with Cool's $V$ magnitude, and the temperature was varied to give the best agreement at $B$ and $I$. The best fit spectrum had a temperature of $22,000 \pm 7,000 \mathrm{~K}$, where the error is $1 \sigma$. Use of this technique means that errors in the continuum determination are dominated by errors in the photometry only (normalization from a separate observation is valid because CG98 have shown that the star does not vary) without incurring larger (unknown) errors by attempting a poorly constrained independent measurement.

Having determined the continuum level, the shape of the $\mathrm{H} \beta$ line for the NF must be determined. Since both the neighboring turnoff star and diffuse light contributions have their own spectral components, estimates of these aperture contaminants are required before the decontaminated $\mathrm{H} \beta$ line profile can be determined (the contribution of the neighboring turnoff star is most important because its relatively hot temperature implies a reasonably strong $\mathrm{H} \beta$ 
line). Therefore, we still made an approximate determination of the telescope pointing during the FOS observations. We compared the flux in the FOS spectrum with the flux derived from a $0.43^{\prime \prime}$ test aperture centered over the NF in the $V$ image from CG98 (thus including all the aperture light, not just that from the NF). We first subtracted away from the original spectrum the earthshine component derived earlier $(V=21.7)$, leaving only constant components. The equivalent $V$ magnitude of this "constant" FOS component is 19.44 mag, in excellent agreement with the $V$ magnitude derived from the WFPC2 image (19.45). Since the NF does not vary and the pointing of HST was effectively constant during the FOS observations (no drifts above the $\sim 0.001^{\prime \prime}$ level), the contamination from the turnoff star in the FOS aperture must be approximately the same as in the test WFPC2 aperture. Using Cool's photometry we decomposed the FOS spectrum into its separate components of NF, turnoff star and diffuse light (all fitted by Kurucz atmospheres). The resulting residual is shown in Figure 5 and the close agreement with zero shows we made a reasonable, self-consistent determination of the various aperture components. Since the residual is so red, its contribution to the $\mathrm{NF} \mathrm{H} \beta$ line profile should be negligible (the source of this residual may be incomplete removal of the earthshine component mentioned earlier).

As noted above, the high temperature and the broad $\mathrm{H} \beta$ line appear consistent with the hypothesis that this star is a high gravity object such as a WD. To test this hypothesis we used the pure hydrogen atmosphere models of Wesemael et al. (1980). Modern refinements of these models do not offer any significant advantages in the analysis of this low $\mathrm{S} / \mathrm{N}$ ratio spectrum. The advantages of using these models are that they include detailed line profiles and cover a large range in $\log \mathrm{g}(4<\log \mathrm{g}<9)$ and $T_{\text {eff }}\left(20,000 \mathrm{~K}<T_{\text {eff }}<150,000 \mathrm{~K}\right)$.

Since the only obvious line present ( $\mathrm{H} \beta$ in absorption) is broader than the emission lines for $\mathrm{CVs} 1-4$, and because the $\mathrm{S} / \mathrm{N}$ ratio in this line is low, we increased the length scale of the smoothing to 11 channels and applied the same smoothing factor to the models. We resampled the Wesemael line profiles to the resolution of the FOS data, applied smoothing and then experimented with different model profile fits (Lorentzians and Voigt profiles gave almost identical results). To fit the line profile the ICFIT algorithm within IRAF/SPLOT was used to fit the continuum and a Lorentzian was used to fit the line profile. The line depth and EW were found to be $0.55 \pm$ 0.05 and $23.9 \pm 2.4 \AA$ respectively, where the errors are a combination of random errors in the parameter measurements (determined by Monte Carlo experiments) and systematic errors in the continuum and line profile measurements.

For simplicity we defined each model $\mathrm{H} \beta$ line with two parameters, the line depth and the EW. We generated a complete grid of these two parameters for all of the available Wesemael models, interpolated the $\log \mathrm{g}=1$ spacing to $\log \mathrm{g}=0.25$ and interpolated the temperature scale where necessary (for some log g values fewer models were available). Since the Wesemael models are for $T_{\text {eff }}>20,000 \mathrm{~K}$, we used the Kurucz models (with $4<\log \mathrm{g}<5$ and $T_{\text {eff }}<20,000 \mathrm{~K}$ ) to extend our line depth/EW grid below 20,000 K down to 10,000 K. (Encouragingly, excellent agreement was found between the Wesemael and Kurucz models in the overlap region of $T_{\text {eff }}=$ $20,000 \mathrm{~K}, 4<\log \mathrm{g}<5$; differences of at most $2 \%$ were found in the two line parameters.) We 
extrapolated the Kurucz models to higher $\log g$ values (from $\log g=5$ to $\log g=9$ ), constrained by the functional form of the Wesemael models for the line depth and EW at 20,000 K, using the same smoothing and interpolation as before (see comments below about the validity of this extrapolation).

Finally, the measured line depth and EW were used to search for a solution in $\log \mathrm{g}$ and $T_{\text {eff }}$. The optimal solution was found to be $T_{\text {eff }}=17,500 \pm 5,000 \mathrm{~K}$ and $\log \mathrm{g}=6.25 \pm 1.0$ ( $1 \sigma$ errors). The large errors in the gravity and temperature are caused by the limited information present in one noisy line, in particular the inability of the spectrum to trace possible narrow line cores. Figure 6 shows the total $\chi^{2}$ for the above solution as a function of $T_{\text {eff }}$ and $\log g$. The optimal solution with $\chi^{2}=0.08$ is marked ("L"). The first, second and fourth contour levels correspond roughly to $1 \sigma, 2 \sigma$ and $3 \sigma$ respectively. Figure 6 also shows the regions where the Wesemael and Kurucz models have been used, and where they were extrapolated. Although extrapolation may incur extra uncertainties, Figure 6 shows that a Wesemael model having $T_{\text {eff }}=20,000$ and $\log \mathrm{g}=6.5$ (with $\chi^{2}=0.8$ ) is close to an optimal solution, without requiring any extrapolation. The Kurucz models are useful because they show that a low gravity solution is probably not consistent with the data (besides independently checking the low temperature, low gravity Wesemael models).

Since the errors for $\log \mathrm{g}$ and $T_{\text {eff }}$ from the $\mathrm{H} \beta$ line measurement are considerable, we briefly discuss extra constraints on these two parameters. First, we note from Figure 6 that relative to the optimal solution ("L") only high temperature/high gravity or low temperature/low gravity solutions are allowed. The high gravity solution with $\log g=7.25$ seems ruled out by the photometry of Cool, Sosin \& King (1997), since the NFs clearly have lower gravities than log g $=7$, the low gravity limit of the models used. The low gravity solution with $\log g=5.25$ and $T_{\text {eff }}=12,500 \mathrm{~K}$ has a temperature that is probably inconsistent with the determination from the photometry of CG98 $(22,000 \pm 7,000 \mathrm{~K})$.

To determine the wavelength of the $\mathrm{H} \beta$ line we adopted a two-step procedure: (1) we used the Lorentzian fit to the $\mathrm{H} \beta$ line as a first-order solution and then (2) used a spectral model (template) with fixed continuum, line depth and EW [determined in (1)] but with variable wavelength to refine the initial estimate. Using template shifts of up to $10 \AA$ in $0.1 \AA$ steps we selected the shift which minimized the difference between the template and measured spectrum. This procedure is formally similar to a cross correlation, but it gives sub-pixel resolution without polynomial fitting and allows us to easily weight the first-order solution to optimize sensitivity to Doppler shifts. Using this procedure the $\mathrm{H} \beta$ line was determined to have a wavelength of $4865.8 \AA$, noticeably redder than the laboratory value of $4861.3 \AA$ [step (1) alone gave $4864.5 \AA$ ]. The random error given by IRAF/SPLOT is only $0.4 \AA$, so we investigated the possibility of systematic effects. According to the HST Data Handbook, the overall $1 \sigma$ random uncertainty is $0.7 \AA$ (or $43 \mathrm{~km} \mathrm{~s}^{-1}$ ) for the accuracy with which the wavelength scale is known in an individual FOS spectrum. However, the possibility of filter grating wheel (FGW) displacements means that a worst-case disagreement in wavelength of over $4 \AA$ is possible unless we have independent confirmation of the wavelength scale (when the wavelength scale is fixed the overall $1 \sigma$ random uncertainty falls to $0.5 \AA$ or $31 \mathrm{~km} \mathrm{~s}^{-1}$ ). 
Our only independent constraints on the wavelength scale are the G570H spectra of CV 4 (obtained over 3 orbits), since no movement of the FGW was made between the observations of the NF and CV 4. The wavelength of the $\mathrm{H} \beta$ line for $\mathrm{CV} 4$ was determined to be $4861.8 \AA$. The $0.5 \AA$ shift from the laboratory value is likely to consist of the velocity of the emission region in the binary, the cluster radial velocity of $21 \mathrm{~km} \mathrm{~s}^{-1}(0.34 \AA)$, orbital motion of the Earth around the Sun, and the motion of the telescope itself (the last 2 effects being negligible given the resolution), plus systematic effects because of the FGW position. While CV emission lines are hardly ideal radial velocity standards in general, we believe that $\mathrm{CV} 4$ provides a useful wavelength reference for several reasons: (1) the measured wavelength for $\mathrm{H} \beta$ is only $0.15 \AA$ red-ward of the laboratory value, taking the cluster radial velocity into account, (2) the $\mathrm{CV} 4 \mathrm{H} \beta$ wavelength measurement appears very stable since the 3 sub-observations for the 3 separate orbits (separated in time by almost 2.5 hours, well over half the expected period of CV 4) give wavelengths of $4861.7 \AA$, $4861.8 \AA$, and $4862.0 \AA$, (3) the line is very symmetrical (unlike CVs 1 and 2) so that the wavelength measurement is unlikely to have been skewed, and (4) the wavelength of $\mathrm{H} \alpha$ for $\mathrm{CV} 4$ is $0.4 \AA$ red-ward of the laboratory value, in excellent agreement with the $0.5 \AA$ red-shift for $\mathrm{H} \beta$. Also, two of the 3 strongest HeI lines give consistent Doppler shifts red-ward of the laboratory value: HeI 4921, shift $=0.35 \pm 0.45 \AA$ and HeI 5876, shift $=0.32 \pm 0.21 \AA$. Only HeI 6678 gives a different shift, $-0.72 \pm 0.25 \AA$ relative to the laboratory value, however this line is over $1800 \AA$ red-ward of $\mathrm{H} \beta$ and therefore has less value as a velocity reference.

Using the wavelength of $\mathrm{H} \beta$ for CV 4 as a reference, the Doppler shift of the NF was measured to be $247 \pm 50 \mathrm{~km} \mathrm{~s}^{-1}$. To derive the error we added together in quadrature the random error $(25$ $\left.\mathrm{km} \mathrm{s}^{-1}\right)$, the systematic instrumental error quoted above $\left(31 \mathrm{~km} \mathrm{~s}^{-1}\right)$ and an estimate of other systematic errors, including the use of CV 4 as a velocity reference $\left(0.5 \AA\right.$ or $\left.31 \mathrm{~km} \mathrm{~s}^{-1}\right)$.

A closeup of the $\mathrm{H} \beta$ line profile is shown in Figure 7. Note, in particular, the vertical lines showing the difference in central wavelengths between the average NF and CV $4 \mathrm{H} \beta$ lines. The significance of the apparent shift of $\mathrm{H} \beta$ for the NF is also clearly shown by comparing the two Lorentzians (the astrophysical significance of this $247 \mathrm{~km} \mathrm{~s}^{-1}$ shift will be discussed in Section 3.2). The variable flux but constant wavelength of the $\mathrm{H} \beta$ line for $\mathrm{CV} 4$ is clearly shown by the 3 emission profiles (the apparently constant velocity will be discussed in a future publication).

\section{Discussion}

\subsection{Cataclysmic variables}

One of the crucial advantages of studying cluster CVs, besides their known distance, is the opportunity to study CVs with different metallicities from those of field CVs (see la Dous, 1991 for examples of UV spectra of field CVs). Although the bluest portion of the G160L spectrum is noisy, the detection of He II $\lambda 1640$ coupled with the non-detection of CIV $\lambda 1550$ is probably a direct reflection of the low metallicity for NGC 6397. The lack of obvious emission at MgII $\lambda 2800$ 
may be another measure of the low cluster metallicity, but we defer detailed spectral modeling incorporating the cluster's low metallicity to a future publication.

We now discuss the significance of the UV distribution of CV 1, the disk brightnesses of CVs 1-4 and their He II line ratios. Regarding the UV flux distribution, one obvious explanation for the redness of the disk explained in Section 2.1, given our sample size of one (for G160L data), is an inclination effect. A second possible explanation is the relatively large contribution from the bright secondary. This explanation seems plausible in that: (1) our predicted period for CV 1 is $5.1 \mathrm{~h}$, while the average period of the 8 DQ Hers in Verbunt's sample is only $3.8 \mathrm{~h}$ (also, PopII secondaries tend to be brighter than PopI secondaries at a given mass - Stehle, Kolb \& Ritter 1997), and (2) if we replaced CV 1 by any one of the other CVs the flux distribution would be much bluer. For example, while the estimated secondary for CV 2 is $\sim 1.3$ mag fainter than the secondary for CV 1, the disk in CV 2 is brighter (in $U_{336}$ ) than the disk in CV 1.

If we then ignore the contribution of the secondary (see Figure 3) a simple interpretation of the flux distribution for CV 1 compared to the field CVs is that it has either: (1) a relatively faint and/or cool disk because of a low accretion rate, or (2) a WD with a moderately strong magnetic field. As noted earlier, AM Her systems (with strong magnetic fields) are not included in Verbunt's sample. These systems, lacking disks, can be quite red, however the likely periods of the NGC 6397 CVs are longer than most AM Her systems (see Ritter \& Kolb 1998). Also, the line emission of the NGC 6397 CVs differs from field AM Her systems (see below).

To investigate (1) we compared $M_{V}$ (disk) and the CV periods from Table 2 with Figures 3.5 and 9.8 of Warner (1995), plots of $M_{V}$ (disk) versus orbital period for non-magnetic field CVs. (The two principal error sources for field CVs are estimates of the secondary component and distance estimates, errors that are considerably reduced by studying cluster CVs). Clearly, the NGC 6397 CVs fall near the faint limit for CV disks when their expected period is considered. Among field CVs only faint DNe in quiescence have disks this faint, and these systems have much longer recurrence times for outbursts than brighter DNe (Warner 1987), possibly explaining the observed paucity of DN outbursts in globular clusters (Shara et al. 1996). Generally CVs with periods above the period gap have disks that are brighter than $M_{V} \sim 8$. Indeed, a large number of observational and theoretical studies have concluded that CVs with periods above the period gap generally have high accretion rates, with correspondingly bright disks while CVs with periods below the period gap have lower accretion rates with fainter disks (Patterson 1984, di Stefano \& Rappaport 1994 and Stehle, Kolb \& Ritter 1997, the latter two studies specifically for PopII CVs).

Another way to emphasize the unusually faint disks of the NGC $6397 \mathrm{CVs}$ is to compare globular cluster and open cluster CVs (minimizing uncertainties in $M_{V}$ (disk) estimates). There are now 6 probable CVs in globular clusters with well-measured VI magnitudes all lying at most $\sim 0.2$ magnitudes away from the MS in the $V$ vs $V-I$ CMD (4 in NGC 6397 and 2 in NGC 6752). We contrast this result with the $3 \mathrm{CVs}$ detected in open clusters that have quiescent $V-I$ colors blue-ward of the MS by $\gtrsim 0.6$ mag (M67; Gilliland et al. 1991), 0.7 mag and 1.0 mag (both 
NGC 6791; Kaluzny et al. 1997). Since M67 and NGC 6791 are much less dense and dynamically evolved than NGC 6397, their CVs are expected to have evolved from primordial binary systems, just as with field CVs. Hence, the detection of a nova-like and Z Cam (relatively high accretion rate DN) system in NGC 6791 is not surprising, since in any sample of field CVs these are among the brightest, intrinsically, because of their high accretion rate (resulting in blue $V-I$ colors). The much lower metallicity of NGC 6397 compared to metal-rich field or open cluster CVs should, in general, mean that the NGC 6397 CVs have higher accretion rates (for given binary parameters), according to Stehle, Kolb \& Ritter (1997), implying that even brighter disks should be present.

A possible explanation we have already suggested (GC95), is that these CVs are mostly magnetic systems with truncated and thus relatively faint disks. Our original suggestion was based on the relative strength of He II $\lambda 4686$ compared to $\mathrm{H} \beta$ for $\mathrm{CVs} 1-3$. To summarise the results in Section 2.3, for the AM Her systems (with their strong magnetic fields), a strong He II $\lambda 4686$ line is a well known feature (Warner 1995). However, known DQ Hers show a large range in He II $\lambda 4686 / \mathrm{H} \beta$ line ratios with values ranging from over one (V533 Her) to zero (AE Aqr). To discriminate between magnetic and non-magnetic systems (and eliminate high accretion systems like nova-likes), Silber (1992) has shown that line ratios of $\mathrm{He}$ II $\lambda 4686 / \mathrm{H} \beta>0.4$ and $\mathrm{EW}(\mathrm{H} \beta)>$ $20 \AA$ are reasonable signatures of magnetic systems.

By combining our knowledge of $M_{V}$ (disk) and the He II line ratios of CVs 1-4, we can attempt identification of these systems. With its weak He II line and faint disk, CV 4 is a reasonable candidate for a quiescent DN system. Since there is remarkable similarity between the He II $\lambda 4686 / \mathrm{H} \beta$ line ratios for CVs $1-3$ and also similarities in the Balmer line fluxes themselves (see Table 1), CVs 1-3 may have very similar properties, as originally suggested by GC95. They do not appear to be recent old novae or nova-likes because of their faint disks (with extra evidence from their He II $\lambda 4686$ line ratios), nor do they appear to be DNe because they have moderately strong He II lines. The final option is magnetic systems. CVs 1-3 do not have He II ratios as large as AM Her systems, but while their ratios are also weaker than an "average" DQ Her system, there is considerable scatter for the DQ Her systems, as pointed out above. For example, CVs 1-3 have He II ratios stronger than 4 of the 8 DQ Her systems in the sample of Williams (1983), and CVs 1 and 2 only just fail the magnetic criteria of Silber (1992). To conclude, CVs 1-3 do not appear to be DNe, but they could be DQ Her type systems.

Is it true that DQ Her systems tend to have disks as faint as those found in CVs 1-4? Extra information about DQ Her disks compared to other CV classes comes from the continuum slopes in Williams (1983). An estimate of the disk contribution (and temperature) relative to the secondary comes from analyzing the continuum ratio between two different wavelengths (equivalent to a color). The two most convenient continuum points for both CVs 1-4 and the spectra of Williams (1983) are at $\mathrm{H} \beta$ and $\mathrm{H} \alpha$. Figure 8 shows plots of this continuum ratio versus the $\mathrm{He} \mathrm{II} / \mathrm{H} \beta$ line ratio for several different classes of field CV (from Williams 1983, updated by Ritter \& Kolb 1998) along with these ratios for CVs 1-4. We examined the linear correlation between the continuum and line ratios for the individual CV classes shown, finding a linear correlation with absolute value 
$>0.5$ in 3 cases: DQ Her systems (linear correlation $=0.77)$, nova-likes $(-0.69)$ and old novae (0.57). The correlation for DQ Hers implies that small He II line ratios imply continuum ratios of $\sim 1.0$ (meaning that the secondary dominates unless the disk is very cool). A notable element of Figure 8 is that all of CVs 1-4 lie close to the best fit line for DQ Hers, and all of them have continuum ratios of $\sim 1.0$. The dominance of the secondary for CVs $1-4$ is therefore exactly what we expect for field DQ Her systems with weak He II lines.

Using estimates of $V$ (disk) and distances for DQ Her systems (from Patterson 1994) we have estimated $M_{V}$ (disk) for DQ Hers, neglecting reddening (the $\sim 50 \%$ errors in the distances dominate the errors) and assuming that the systems are in their faint, low accretion state. We found a strong linear correlation $(-0.83)$ between the $\mathrm{He} \mathrm{II} / \mathrm{H} \beta$ line ratio and $M_{V}$ (disk), as shown in the upper panel of Figure 8, in the sense that higher $\mathrm{He} \mathrm{II} / \mathrm{H} \beta$ implies a brighter disk (consistent with the bluer colors given above). We then used this correlation to predict $M_{V}$ (disk) using the measured $\mathrm{He} \mathrm{II} / \mathrm{H} \beta$ line ratios for CVs 1-4 given in Section 2.2. The results are shown in the final column of Table 2, and agree nicely with the $M_{V}$ (disk) values given in Table 2 for CVs 1-4, especially given the large uncertainties in $M_{V}$ for the field CVs. (We excluded GK Per from the sample, with its $\sim 2$ day period, evolved companion and thus high accretion rate. Including GK Per gave a linear correlation of -0.59 between $M_{V}$ (disk) and $\mathrm{He} \mathrm{II} / \mathrm{H} \beta$ and $M_{V}$ (disk) values $\sim 0.6$ mag brighter.) These results are the best evidence that these cluster CVs are indeed largely DQ Her type systems.

A possible alternative to the DQ Her hypothesis is that some of the $6397 \mathrm{CV}$ s are old novae (possibly in deep hibernation between outbursts; see Shara et al. 1986). This explanation is consistent with the correlation noted above between the continuum ratios and $\mathrm{He} \mathrm{II} / \mathrm{H} \beta$ line ratios for old novae, plus the proximity of the $6397 \mathrm{CVs}$ to the best-fit line for old novae. Although the hibernation scenario is difficult to test (and yet to be confirmed), upcoming HST spectra of the probable old nova in M80 will provide a critical comparison with the spectra of the NGC 6397 CVs. It is also possible that some of the cluster CVs fall in both categories, since, for example, 3 of the DQ Hers in the sample from Williams (1983) are also old novae.

\subsection{Helium White Dwarfs}

The photometry of the NFs (CG98) and the spectroscopy presented above place important constraints on the possible properties and evolution of these systems, which appear to be helium WDs. From the spectroscopy we have already determined $T_{\text {eff }}$ and $\log \mathrm{g}$ and from the photometry we calculated the luminosity of the $\mathrm{NF}\left(\log \left(L / L_{\odot}\right)=-0.6\right)$ using the bolometric corrections presented in Bergeron, Wesemael \& Beauchamp (1995). Armed with these three quantities we have examined evolutionary models of WDs to estimate masses and lifetimes for the NFs. We began by using the WD evolutionary models presented by Bergeron et al. (1995). Models with $\log \mathrm{g}=7$ (the limit of Bergeron's models), $T_{\text {eff }}=17,500 \mathrm{~K}$ and with thick hydrogen layers have $M \approx 0.3 M_{\odot}$. Extrapolating to $\log \mathrm{g}=6.25$, models should have $M \approx 0.2-0.25 M_{\odot}$. Using the 
study by Webbink (1975) of the evolution of helium WDs in close binaries, we used the measured $\log \left(L / L_{\odot}\right)$ and $T_{\text {eff }}$ to derive a mass between 0.2 and $0.25 M_{\odot}$ for the NF, with cooling ages for these models between $\sim 2 \times 10^{8} \mathrm{yr}\left(0.25 M_{\odot}\right)$ and $\sim 5 \times 10^{8} \mathrm{yr}\left(0.20 M_{\odot}\right)$. Using $\log \mathrm{g}, T_{\text {eff }}$ and $\log \left(L / L_{\odot}\right)$, a similar mass is determined from the He WD study of Althaus \& Benvenuto (1997), although they do not include a hydrogen envelope.

Since the only significant line present in the NF is $\mathrm{H} \beta$, a hydrogen envelope must be present, and therefore we have made comparisons with the models of Benvenuto \& Althaus (1998) on the effects of hydrogen envelopes on the structure and evolution of low- and intermediate-mass WDs. They computed the evolution of WDs with masses from $0.15-0.5 M_{\odot}$, while treating the mass of the hydrogen envelope as a free parameter within the range $10^{-8} \leq M_{H} / M \leq 4 \times 10^{-3}$. Because only a representative sample of the models are presented in Benvenuto \& Althaus (1998), L. Althaus has kindly performed a dedicated search for models consistent with our $\log g$ and $T_{\text {eff. }}$. Two good solutions were found for a $0.24 M_{\odot}$ model with $M_{H} / M=1 \times 10^{-3}$ and a $0.235 M_{\odot}$ model with $M_{H} / M=5 \times 10^{-4}$, with both of these solutions having $\log \left(L / L_{\odot}\right)=-0.52$, in excellent agreement with the measured luminosity. These solutions are close to the valid bright limit of the models of Benvenuto \& Althaus (1998), so the cooling age for these solutions of $\sim 7 \times 10^{7}$ yr is highly uncertain (and likely to be an underestimate) since Benvenuto \& Althaus (1998) did not attempt a detailed treatment of the formation of helium WDs in binary systems.

A detailed treatment of the evolution of a $0.3 M_{\odot}$ WD in a binary system was given by Iben \& Tutukov (1986; hereafter IT86). Their model experiences two hydrogen shell flashes before cooling to $T_{\text {eff }} \sim 16,000 \mathrm{~K}$ at an age of $\sim 1 \times 10^{8} \mathrm{yr}$ (a comparable age to that found by Benvenuto \& Althaus (1998), but smaller than Webbink's values). Using their cooling curve, IT86 construct a number-luminosity distribution function for helium WDs. This function has a similar slope to that for CO WDs, but at most magnitudes is about a factor of 4 smaller. One exception to this behavior is the region with $-1 \lesssim \log \left(L / L_{\odot}\right) \lesssim-1.7$. At $\log \left(L / L_{\odot}\right) \sim-1$ the predicted helium WD number-luminosity function shows a "bump" where it increases to be roughly equal to the CO WD function. It then drops quickly with decreasing luminosity by about an order of magnitude before returning to the average value at $\log \left(L / L_{\odot}\right) \sim-1.7$. This behavior is caused by the very slow rate of decline in luminosity following the first hydrogen shell flash, when $t=10^{7}-10^{8}$ yr. Although IT86 warn that a complete theoretical distribution function requires superposition of contributions from WDs of many different masses (each experiencing a hydrogen shell flash at a different luminosity), this oscillatory behavior may cause an enhancement in the number of helium WDs at relatively high luminosity and a dearth for $\sim 1.5$ mag below this. This could be consistent with the observed CMD distribution in CG98, perhaps explaining the lack of obvious low-mass WDs further down a cooling sequence, particularly since $\log \left(L / L_{\odot}\right) \sim-1$ is not far from the measured luminosities for the two faintest helium WD candidates (eventually close double degenerate systems should merge after a few Gyr, forming a more massive WD). However, clearly more data from other clusters are needed to test this hypothesis.

An obvious question remains: are the possible formation mechanisms listed in Section 1 
consistent with the likely significant red-shift of the $\mathrm{H} \beta$ line? We reject the possibility of the red-shift being an ejection velocity, since at $247 \mathrm{~km} \mathrm{~s}^{-1}$, the star would have moved $2.2 \mathrm{pc}$ in only $10^{4} \mathrm{yr}$, and thus would be well outside the core. Gravitational red-shift is likely to contribute only a small red-shift for this low-mass object, since $K_{R}=0.635 \times M / M_{\odot} \times R_{\odot} / R \mathrm{~km} \mathrm{~s}^{-1}$ (Reid 1996), and using the mass and radius estimates from Benvenuto and Althaus' models, $K_{R} \approx 3 \mathrm{~km} \mathrm{~s}^{-1}$. Instead, we argue that the red-shift may be a Doppler shift from the helium WD orbiting a massive but faint companion, probably a WD. This is consistent with the primary formation mechanism listed above. Also, a binary nature for this star would hardly be surprising for dynamical reasons alone, since the detection of 3 NFs near the center of NGC 6397 is good evidence of a binary origin for these stars (through mass segregation), and most known low-mass WDs are in binary systems. Finally, we note the possibility that these low-mass WDs may have neutron star companions, likely to be binary millisecond pulsars (although none have been found yet in NGC 6397) as found in helium WD/NS binary systems in the field.

If we have measured an orbital Doppler shift its size is an important consideration. Of the 8 double-degenerate systems listed in Iben et al. (1997), all with a helium WD as the primary (the brighter component), only 2 have $K>150 \mathrm{~km} \mathrm{~s}^{-1}$. Little is known about the masses of the secondaries in these systems, but models by Iben et al. (1997) show that $K \approx 250 \mathrm{~km} \mathrm{~s}^{-1}$ should be quite common for high-inclination systems with a helium WD primary and a CO WD secondary (from Cool, Piotto \& King 1996 and CG98, NGC 6397 clearly has a large reservoir of $\sim 0.55 M_{\odot} \mathrm{CO}$ WDs, and it should also have many higher mass WDs). For more massive secondaries such as neutron stars, larger values of $K$ can be found, for example $K=280 \mathrm{~km} \mathrm{~s}^{-1}$ for the helium WD - MSP binary J1012+5307 (van Kerkwijk, Bergeron \& Kulkarni 1996).

Adopting a range of possible mass functions for a binary system containing the NF and a unknown companion, and assuming $K=247 \mathrm{~km} \mathrm{~s}^{-1}$ and helium WD mass $=0.25 M_{\odot}$, we have derived expected periods for the system as a function of orbital inclination $(i)$. Although the NF observations were spread over 3 (96 minute) orbits, $\sim 42$ min of spectra were taken on the middle orbit, but only $\sim 10$ min at the end of the first orbit and $\sim 14$ min at the start of the third. Therefore, we cannot rule out a $\sim 4-5 \mathrm{~h}$ period, and for $i<60^{\circ}$ we require a WD companion with mass $\gtrsim 0.7 M_{\odot}$, slightly higher than the mass of WDs currently being produced in the cluster (low-mass MS stars are ruled out, consistent with the photometry of CG98). Since we have measured only a lower limit for $K$, we have also experimented with values that are $25 \%$ higher than observed, maintaining the other assumptions. In this case a high mass WD (mass $\gtrsim$ $1.1 M_{\odot}$ ) or neutron star companion is required (clearly longer observations may provide powerful constraints on the mass of the companion).

To determine whether 3 systems with ages of $\sim 1-5 \times 10^{8}$ yr (IT86 and Webbink 1975) are likely to be present in NGC 6397, we used expected encounter rates between various combinations of MS stars, WDs, neutron stars and red giants (Davies \& Benz 1995) to make rough estimates of expected numbers of binaries or merger products. The calculations by Davies \& Benz (1995) are specifically for $\omega$ Cen and 47 Tuc - we used their numbers for the denser cluster 47 Tuc and 
divided the interaction rates by 5 to account for the smaller mass of NGC 6397 (using cluster masses from Pryor \& Meylan, 1993). Assuming a lifetime of 4 Gyr (Sandquist, Bolte, \& Hernquist 1997) for blue stragglers less than $\sim 1.5$ mag brighter than the MS turnoff (where most of the observed blue stragglers are found) and assuming all MS star collisions result in mergers, we used the calculations of Davies \& Benz (1995) to estimate that 75 blue stragglers should currently be found in NGC 6397 (from both 2- and 3-body interactions), comparing favorably with the 54 blue straggler candidates found by Kaluzny (1997). An overestimate of total blue stragglers is not surprising because merged stars with total masses at or below the turnoff are, of course, not included in Kaluzny's sample. For CVs, we assumed that half the collisions between WDs and MS stars result in the formation of binaries (Davies \& Benz 1995), and used a conservative limit on the mass ratio of 1 for stable mass transfer (Davies \& Benz 1995). Then, assuming the average CV lifetime is 1.5 Gyr for CVs above the period gap (Kolb \& Stehle 1996), we expect 6 CVs from 2 - and 3-body interactions, close to the observed number. Finally, assuming (1) ages of $0.1-0.5$ Gyr for the observed helium WDs, (2) that all of the red giant collisions with either neutron stars or WDs result in the formation of He WDs (see Davies, Benz \& Hills 1991), and (3) that 3-body interactions result in as many helium WDs as 2-body interactions, we predict $0.7-3.5$ bright helium WDs to currently be found in NGC 6397.

The reasonable agreement between observed and estimated numbers of blue stragglers and $\mathrm{CVs}$ is comforting given the large uncertainties in the interaction rates used, the influence of possible differences in interaction rates between NGC 6397 and 47 Tuc (e.g. NGC 6397 is core-collapsed and 47 Tuc may or may not be), and the effect of the unknown binary content (Davies \& Benz 1995 assume 10\% binaries). Since the lifetimes for blue stragglers are known reasonably well and the number of observed systems is probably fairly complete, the possible differences listed above must either be small or cancel each other out. We also have reasonable agreement between the numbers of expected and observed helium WDs when using Webbink's cooling ages. Although our assumptions may appear somewhat generous, there may be other mechanisms for the formation of He WDs: for example, red giant - MS star collisions, binary binary collisions and primordial binary evolution.

It is the expected lifetimes for the helium WDs that are one of the largest sources of uncertainty in the above number estimates. The (unknown) thickness of the hydrogen envelope can make a significant difference to the lifetimes especially if hydrogen burning occurs. For example, a model with $M=0.3 M_{\odot}$ and $M_{H} / M=2 \times 10^{-3}$ (the thickest envelope considered by Benvenuto \& Althaus (1998), with $\log g$ and $T_{\text {eff }}$ consistent with our low-mass NF within the errors) has an age $\sim 50 \%$ greater than the $0.3 M_{\odot}$ model with $M_{H} / M=1 \times 10^{-3}$. It has also been suggested (Alberts et al. 1996) that the cooling time-scales of very low-mass WDs (mass < $\left.0.25 M_{\odot}\right)$ can be considerably underestimated by the traditional WD cooling curves of IT86 and others for higher mass WDs (mass $>0.3 M_{\odot}$ ). Alberts et al. (1996) predict that hydrogen shell flashes do not occur on WDs with mass $<0.2 M_{\odot}$, but that these WDs experience long-lasting phases of hydrogen burning which significantly prolong their lifetimes (however this difference in 
behavior may be caused by differences in time resolution between the models of Alberts et al. and IT86). Finally, Sarna, Antipova \& Muslimov (1998) find much greater cooling ages for their $0.16 M_{\odot}$ WD compared to IT86 because of differences in the formation mechanisms. While in IT86 the donor star fills its Roche lobe when it is on the red giant branch, forming a $\sim 0.3 M_{\odot}$ helium WD with a relatively thin hydrogen envelope, the donor star in Sarna et al.'s calculations fills its Roche lobe while it is evolving through the Hertzsprung gap, resulting in a $0.16 M_{\odot}$ WD with a much thicker hydrogen envelope.

Since bright red giants have much shorter lifetimes than subgiants (and perhaps limited cross-sections, despite larger radii, because of low densities) it is possible that collisions involving subgiants or faint red giants are much more efficient at producing helium WDs than collisions involving brighter red giants. This selection effect would preferentially cause very low-mass $\left(\lesssim 0.25 M_{\odot}\right)$ WDs to form (since the red giant core mass increases with increasing brightness), giving objects with longer cooling times according to Webbink (1975) and Sarna et al. (1998), and enhancing the number of helium WDs seen.

\section{Conclusion}

We summarize here the results for CVs 1-4: (1) a 4th CV candidate in NGC 6397 has been spectroscopically confirmed, (2) UV data for CV 1 implies that it has a red disk when compared with field CVs, (3) the photometry of CG98 combined with Kurucz spectra for the estimated secondaries provide strong evidence that CVs 1-4 all have faint disks and probably low accretion rates (consistent with faint quiescent $\mathrm{DNe}$ ), (4) the $\mathrm{He}$ II $\lambda 4686 / \mathrm{H} \beta$ line ratios (together with the faint disks) imply that CVs 1-3 may be DQ Her systems, (5) the correlations between the $\mathrm{He} \mathrm{II} / \mathrm{H} \beta$ line ratios for CVs $1-4$ and both (a) the continuum ratios between $\mathrm{H} \beta$ and $\mathrm{H} \alpha$ and (b) $M_{V}$ (disk) provide extra evidence that the $6397 \mathrm{CVs}$ are mainly DQ Her systems. This is consistent with the finding of Verbunt et al. (1997) that CVs 1-3 could be DQ Her systems based on their X-ray and optical fluxes. An alternative explanation is that some of the CVs are old novae in hibernating phases between nova eruptions.

We conclude that there may be fundamental differences between populations of globular cluster CVs and field/open cluster CVs, perhaps caused by the different formation mechanism of tidal capture and exchange collisions or perhaps because of the different environment in globular clusters. One possible explanation is that interactions cause stars to rotate more quickly, resulting in stronger magnetic fields than in most field stars, as suggested by GC95. Alternatively, Vandenberg, Larson \& De Propris (1998) have suggested that rapidly rotating cores of giant stars in the metal poor globular cluster M30 might reconcile this cluster's luminosity function with stellar evolutionary theory. Similar problems exist in understanding the luminosity function of NGC 6397, although further study of the theory of Vandenberg et al. (1998) is needed. Another possibility is that magnetic WDs are formed in globulars from differentially rotating cores in blue stragglers (Grindlay 1996). 
Prospects for further work on the CV data include modeling of the disk for CV 1, power spectrum analysis of both the time series obtained by CG98 and the sub-exposures for the FOS spectra, studies of line profile changes with time and detailed spectral modeling incorporating the cluster's low metallicity. A clear test of the hypothesis that most of the 4 CVs are DQ Her systems is to search for a stable optical (or X-ray) period with $P<P_{\text {orb }}$ (DQ Hers usually have $P \ll P_{\text {orb }}$; Patterson 1994). Because the short FOS observations of the CVs are inadequate for this purpose, we shall propose to obtain simultaneous spectra of CVs 1 and 2 using moderate time-resolution ( $\Delta \tau \sim 60$ s) spectroscopy in the blue (using STIS with a long slit), to directly test the magnetic $\mathrm{CV}$ hypothesis and place constraints on the hibernating nova scenario.

The results for the NF are: (1) using detailed comparisons with stellar atmospheres from Wesemael et al. (1980) and Kurucz (1993) we have determined $\log \mathrm{g}=6.25 \pm 1.0$, and $T_{\text {eff }}=$ $17,500 \pm 5,000 \mathrm{~K}$ (consistent with $T_{\text {eff }}=22,000 \pm 7,000 \mathrm{~K}$ using the photometry of CG98), (2) by using these line parameters and the luminosity of the NF we have shown that the NF spectrum is consistent with a helium WD having a mass of $\sim 0.25 M_{\odot}$ and an age between 0.1 and 0.5 Gyr (depending on the models used), and (3) the NF spectrum appears to be significantly Doppler shifted from the expected wavelength, suggesting the presence of a dark, massive companion. The low mass of the NF (and probably similar or lower masses for the others) suggest that interactions between degenerate stars and subgiants or faint red giants are more efficient at producing helium WDs than interactions involving degenerate stars and brighter red giants.

Although we have not yet made a rigorous attempt to find evidence for velocity variability of the $\mathrm{H} \beta$ line for the $\mathrm{NF}$, the prospects from subdividing this low $\mathrm{S} / \mathrm{N}$ spectrum are poor, especially since almost two thirds of the data for the NF was obtained over just one $\sim 42$ min time segment. Clearly, observations over a longer time are needed to confirm that Doppler shift evidence presented above and to study radial velocity variations. Use of STIS with the long slit would enable two NFs to be observed simultaneously, along with many cluster stars providing an ideal radial velocity reference. Observations in the blue would also give excellent coverage of Balmer absorption lines (with the exception of $\mathrm{H} \alpha$ ), giving considerably more accurate line parameters, and helping determine whether the luminosity difference between the bright NF and the two fainter ones is mainly because of mass or age differences.

We thank R. Kurucz, L. Althaus and O. Benvenuto for models, B. Hansen, R. Di Stefano and F. Wesemael for discussions and an anonymous referee for helpful comments. This work was partially supported by NASA grants NAG5-3808 and HST grant GO-06742 (PDE and JEG), and NASA grant NAG5-6404 (CDB). 


\section{REFERENCES}

Alberts, F., Savonije, G. J., van den Heuvel, E. P. J. \& Pols, O. R. 1996, Nature, 380, 676

Alcaino, G., Liller, W., Alvarado, F., Kravtsov, V., Ipatov, A., Samus, N. \& Smirnov, O. 1997, AJ, 114, 1067

Althaus, L. G. \& Benvenuto, O. G. 1997, ApJ, 477, 313

Anderson, S. F., Margon, B., Deutsch, E. W. \& Downes, R. A. 1993, AJ, 106, 1049

Anderson, S. F., Margon, B., Deutsch, E. W., Downes, R. A. \& Allen, R. G. 1997, ApJ, 482, L69

Bailyn, C. D. \& Grindlay, J. E. 1987, ApJ, 316, L25

Bailyn, C. D., Rubenstein, E. P., Slavin, S. D., Cohn, H. N., Lugger, P. M., Cool, A. M. \& Grindlay, J. E. 1996, ApJ, 473, L31

Benvenuto, O. G. \& Althaus, L. G. 1998, MNRAS, 293, 177

Bergbusch, P. A. \& Vandenberg, D. A. 1992, ApJS, 81, 163

Bergeron, P., Wesemael, F. \& Beauchamp, A. 1995, PASP, 107, 1047

Cool, A. M., Grindlay, J. E., Krockenberger, M., \& Bailyn, C. D. 1993, ApJ, 410, L103

Cool, A. M., Grindlay, J. E., Cohn, H. N., Lugger, P. M., \& Slavin, S. D. 1995, ApJ, 439, 695

Cool, A. M., Piotto, G. \& King, I. R. 1996, ApJ, 468, 655

Cool, A. M., Sosin, C. \& King, I. R. 1997, in White Dwarfs, eds J. Isern, M. Hernanz and E. Garcia-Berro (Dordrecht: Kluwer Academic Publishing), p. 129

Cool, A. M., Grindlay, J. E., Cohn, H. N., Lugger, P. M. \& Bailyn, C. D. 1998, ApJ, submitted (CG98)

Davies, M. B. 1997, MNRAS, 288, 117

Davies, M. B., \& Benz, W. 1995, MNRAS, 276, 876

Davies, M. B., Benz, W. \& Hills, J. G. 1991, ApJ, 381, 449

Di Stefano, R. \& Rappaport, S. 1994, ApJ, 423, 274

Echevarria, J. 1988, ApJ, 233, 513

Edmonds, P. D., Gilliland, R. L., Guhathakurta, P., Petro, L. D., Saha, A., \& Shara, M. M. 1996, ApJ, 468, 241

Gilliland, R. L., Brown, T. M., Duncan, D. K., Suntzeff, N. B., Lockwood, G. W., Thompson, D. T., Schild, R. E., Jeffrey, W. A. \& Penprase, B.E. 1991, AJ, 101, 541

Grindlay, J. E., 1996, in Proceedings IAU Symposium 174 (eds J. Makino \& P. Hut), 171

Grindlay, J. E., 1998, Proc. Anapolis Conf. on Magnetic CVs (eds C. Hellier and K. Mukai), ASP Conf. Series, in press 
Grindlay, J. E., Cool, A. M., Callanan, P. J., Bailyn, C. D., Cohn, H. N. \& Lugger, P. M. 1995, ApJ, 455, L47 (GC95)

Hertz, P. \& Grindlay, J. E. 1983, ApJ, 275, 105

Hut, P., McMillan, S., Goodman, J., Mateo, M., Phinney, E. S., Pryor, C., Richer, H. B., Verbunt, F., \& Weinberg, M. 1992, PASP, 104, 981

Iben, I.Jr. \& Tutukov, A. V. 1986, ApJ, 311, 742 (IT86)

Iben, I.Jr., Tutukov, A. V. \& Yungelson, L.R. 1997, ApJ, 475, 291

Kaluzny, J. 1997, A\&AS, 122, 1

Kaluzny, J., Stanek, K. Z., Garnavich, P. M. \& Challis, P. 1997, ApJ, 491, 153

Kaluzny, J., Kubiak, M., Szymanski, M., Udalski, A., Krzeminski, W., Mateo, M. \& Stanek, K. Z. 1998, A\&AS, 128, 19

Kolb, U. \& Stehle, R. 1996, MNRAS, 282, 1454

Kurucz, R.L. 1993, ATLAS9 Stellar Atmosphere Programs and $2 \mathrm{~km} \mathrm{~s}^{-1}$ grid (Kurucz CD-ROM No. 13)

la Dous, C. 1991, A\&A, 252, 100

Landsman, W., Aparicio, J., Bergeron, P., Di Stefano, R. \& Stecher, T.P. 1997, ApJ, 481, L93

Manchester, R. N., Lyne, A. G., Robinson, C., D’Amico, N., Bailes, M., \& Lim, J. 1991, Nature, 352,219

Marsh, T. R., Dhillon, V. S. \& Duck, S.R. 1995, MNRAS, 275, 828

Oosterhoff, P., 1941, Ann. Sterrewacht Leiden, 17 No. 4

Paresce, F. \& De Marchi, G. 1994, ApJ, 427, L33

Patterson, J. 1984, ApJS, 54, 443

Patterson, J. 1994, PASP, 106, 209

Pryor, C. \& Meylan, G. 1993, in Structure and Dynamics of Globular Clusters, ed. S.G. Djorgovski \& G. Meylan (ASP Conf. series), 357

Rappaport, S., Podsiadlowski, P., Joss, P. C., Di Stefano, R. \& Han, Z. 1995, MNRAS, 273, 731

Reid, I. N. 1996, AJ, 111, 2000

Ritter, H. \& Kolb, U. 1998, A\&AS, 129, 83

Rubenstein, E. P. \& Bailyn, C. D. 1997, ApJ, 474, 701

Sandquist, E. L., Bolte, M. \& Hernquist, L. 1997, ApJ, 477, 335

Sarna, M. J., Antipova, J. \& Muslimov, A. 1998, ApJ, 499, 407

Shara, M. M., Livio, M., Moffat, A. F. J. \& Orio, M. 1986, ApJ, 311, 163

Shara, M. M., Bergeron, L.E., Gilliland, R. L., Saha, A. \& Petro, L. D. 1996, ApJ, 471, 804 
Shara, M. M. \& Drissen, L. 1995, ApJ, 448, 203

Shara, M. M., Zurek, D. R. \& Rich, R. M. 1996, ApJ, 473, L35

Silber, A. 1992, Ph.D. thesis, MIT

Smith, D. A. \& Dhillon, V. S. 1998, MNRAS, in press

Stehle, R., Kolb, U. \& Ritter, H. 1997, A\&A, 320, 136

Vandenberg, D. A., Larson, A. M. \& De Propris, R. 1998, PASP, 110, 98

van Kerkwijk, M. H., Bergeron, P. \& Kulkarni, S. R. 1996, ApJ, 467, L89

Verbunt, F. 1987, A\&AS, 71, 339

Verbunt, F., Bunk, W. H., Ritter, E. \& Pfefferman, E. 1997, A\&A, 327, 602

Warner, B. 1987, MNRAS, 227, 23

Warner, B. 1995, Cataclysmic Variable Stars, Cambridge University Press

Webbink, R. F. 1975, MNRAS, 171, 555

Wesemael, F., Auer, L. H., Van Horn, H. M. \& Savedoff, M. P. 1980, ApJS, 43, 159

Williams, G. 1983, ApJS, 53, 523 
Table 1. Emission line data

\begin{tabular}{|c|c|c|c|c|c|}
\hline & $\begin{array}{l}\text { Wavelength } \\
(\AA)\end{array}$ & Flux ${ }^{a}$ & $\begin{array}{l}\text { EW } \\
(\AA)\end{array}$ & $\begin{array}{c}\text { Gaussian }^{\mathrm{b}} \\
\text { FWHM } \\
(\AA)\end{array}$ & $\begin{array}{c}\text { Lorentzian }^{\mathrm{b}} \\
\text { FWHM } \\
(\AA)\end{array}$ \\
\hline \multicolumn{6}{|c|}{ CV 1} \\
\hline He II & $4687.04 \pm 0.59$ & $1.26 \pm 0.11$ & $5.9 \pm 0.5$ & $21.0 \pm 2.0$ & $0.0 \pm 2.7$ \\
\hline $\mathrm{H} \beta$ & $4858.09 \pm 0.21$ & $3.92 \pm 0.16$ & $18.0 \pm 0.8$ & $3.8 \pm 5.7$ & $15.6 \pm 1.6$ \\
\hline He I & $5872.91 \pm 0.45$ & $1.51 \pm 0.14$ & $6.9 \pm 0.7$ & $21.6 \pm 2.2$ & $0.0 \pm 2.7$ \\
\hline $\mathrm{H} \alpha$ & $6559.19 \pm 0.30$ & $4.40 \pm 0.18$ & $21.0 \pm 0.9$ & $24.0 \pm 1.2$ & $0.0 \pm 1.6$ \\
\hline \multicolumn{6}{|c|}{$\mathrm{CV} 2$} \\
\hline He II & $4688.37 \pm 0.86$ & $1.44 \pm 0.16$ & $11.2 \pm 1.3$ & $13.9 \pm 12.0$ & $23.6 \pm 7.1$ \\
\hline $\mathrm{H} \beta$ & $4862.47 \pm 0.17$ & $4.19 \pm 0.15$ & $31.9 \pm 1.2$ & $20.8 \pm 2.0$ & $11.1 \pm 2.1$ \\
\hline He I & $5873.98 \pm 0.53$ & $1.04 \pm 0.10$ & $7.6 \pm 0.8$ & $21.7 \pm 2.8$ & $0.0 \pm 3.2$ \\
\hline $\mathrm{H} \alpha$ & $6563.60 \pm 0.39$ & $3.94 \pm 0.16$ & $30.2 \pm 1.3$ & $30.7 \pm 1.5$ & $2.1 \pm 2.1$ \\
\hline \multicolumn{6}{|c|}{ CV 3} \\
\hline He II & $4685.71 \pm 0.81$ & $0.53 \pm 0.05$ & $15.2 \pm 1.5$ & $26.2 \pm 5.5$ & $10.6 \pm 7.3$ \\
\hline $\mathrm{H} \beta$ & $4860.73 \pm 0.12$ & $2.07 \pm 0.05$ & $59.0 \pm 1.6$ & $13.8 \pm 0.9$ & $10.5 \pm 0.8$ \\
\hline He I & $5874.59 \pm 0.37$ & $0.53 \pm 0.03$ & $13.6 \pm 1.0$ & $18.6 \pm 1.5$ & $0.0 \pm 1.6$ \\
\hline $\mathrm{H} \alpha$ & $6562.94 \pm 0.24$ & $2.81 \pm 0.09$ & $71.7 \pm 2.8$ & $3.1 \pm 4.1$ & $20.2 \pm 1.4$ \\
\hline \multicolumn{6}{|c|}{ CV 4} \\
\hline He II & $4685.60 \pm 0.67$ & $0.20 \pm 0.03$ & $12.3 \pm 1.8$ & $21.0 \pm 3.6$ & $0.0 \pm 4.1$ \\
\hline $\mathrm{H} \beta$ & $4861.84 \pm 0.05$ & $2.73 \pm 0.03$ & $158.0 \pm 2.7$ & $9.4 \pm 0.5$ & $8.1 \pm 0.4$ \\
\hline He I & $5874.78 \pm 0.18$ & $0.79 \pm 0.03$ & $29.3 \pm 1.4$ & $15.0 \pm 0.8$ & $0.0 \pm 1.0$ \\
\hline $\mathrm{H} \alpha$ & $6563.27 \pm 0.06$ & $3.39 \pm 0.04$ & $106.7 \pm 1.7$ & $11.9 \pm 0.3$ & $6.2 \pm 0.4$ \\
\hline
\end{tabular}

a The flux is in units of $10^{-15} \mathrm{ergs} \mathrm{cm}^{-2} \mathrm{~s}^{-1} \AA^{-1}$

${ }^{\mathrm{b}}$ The Gaussian and Lorentzian components combine to give the Voigt profile

Note. - The $1 \sigma$ errors quoted here have been calculated by Monte Carlo simulations within SPLOT based on the photon noise errors only. Systematic errors not included here are: (1) $1 \sigma$ wavelength errors of $\sim 0.76 \AA$ (due mainly to FGW errors) and (2) 10-20\% errors in the EWs because of near neighbour and sky contamination (see text). 
Table 2. Absolute magnitudes of CV secondaries and disks

\begin{tabular}{|c|c|c|c|c|c|c|c|c|}
\hline $\mathrm{CV}$ & $\begin{array}{c}M_{V} \\
\text { (total) }\end{array}$ & $\begin{array}{c}M_{V} \\
\text { (secondary) }\end{array}$ & $\begin{array}{l}\text { Mass }\left(M_{\odot}\right)^{\mathrm{a}} \\
(\text { secondary) }\end{array}$ & $\begin{array}{l}\text { predicted }^{\mathrm{b}} \\
\text { period }(\mathrm{h})\end{array}$ & $\begin{array}{c}M_{V} \\
(\text { disk })\end{array}$ & $\begin{array}{r}M_{V}{ }^{\mathrm{c}} \\
(\text { disk })\end{array}$ & $\begin{array}{r}M_{V}{ }^{\mathrm{d}} \\
(\text { disk })\end{array}$ & $\begin{array}{r}M_{V}^{\mathrm{e}} \\
(\mathrm{HeII})\end{array}$ \\
\hline $\mathrm{CV} 1$ & 5.95 & 5.93 & 0.68 & 5.1 & $\ldots$ & 8.5 & 7.6 & 8.5 \\
\hline CV 2 & 7.13 & 7.24 & 0.57 & 4.4 & 9.7 & 8.7 & 8.2 & 8.5 \\
\hline CV 3 & 7.84 & 8.18 & 0.49 & 3.8 & 9.3 & 8.9 & 8.8 & 8.7 \\
\hline $\mathrm{CV} 4$ & 8.41 & 8.53 & 0.45 & 3.6 & 10.9 & 9.9 & 9.9 & 9.3 \\
\hline
\end{tabular}

aThe secondary masses are from Bergbusch and Vandenberg (1992) using the estimated $M_{V}$ values, and differ from those derived by CG98, using different stellar evolution models, by at most only 0.01 $M_{\odot}$.

${ }^{\mathrm{b} U s i n g}$ Warner (1995) and Stehle, Kolb \& Ritter (1997).

${ }^{\mathrm{c}}$ After making the estimated $I$ magnitude of the secondary fainter by 0.1 mag

${ }^{\mathrm{d}}$ After making the estimated $I$ magnitude of the secondary fainter by $0.2 \mathrm{mag}$

ePredicted $M_{V}$ using the HeII $\lambda 4686$ line ratios given in Section 2.2 and the He II $\lambda 4686 / M_{V}$ correlation discussed at the end of Section 3.1.

Note. - We have assumed an apparent distance modulus of 12.28 (from CG98) 


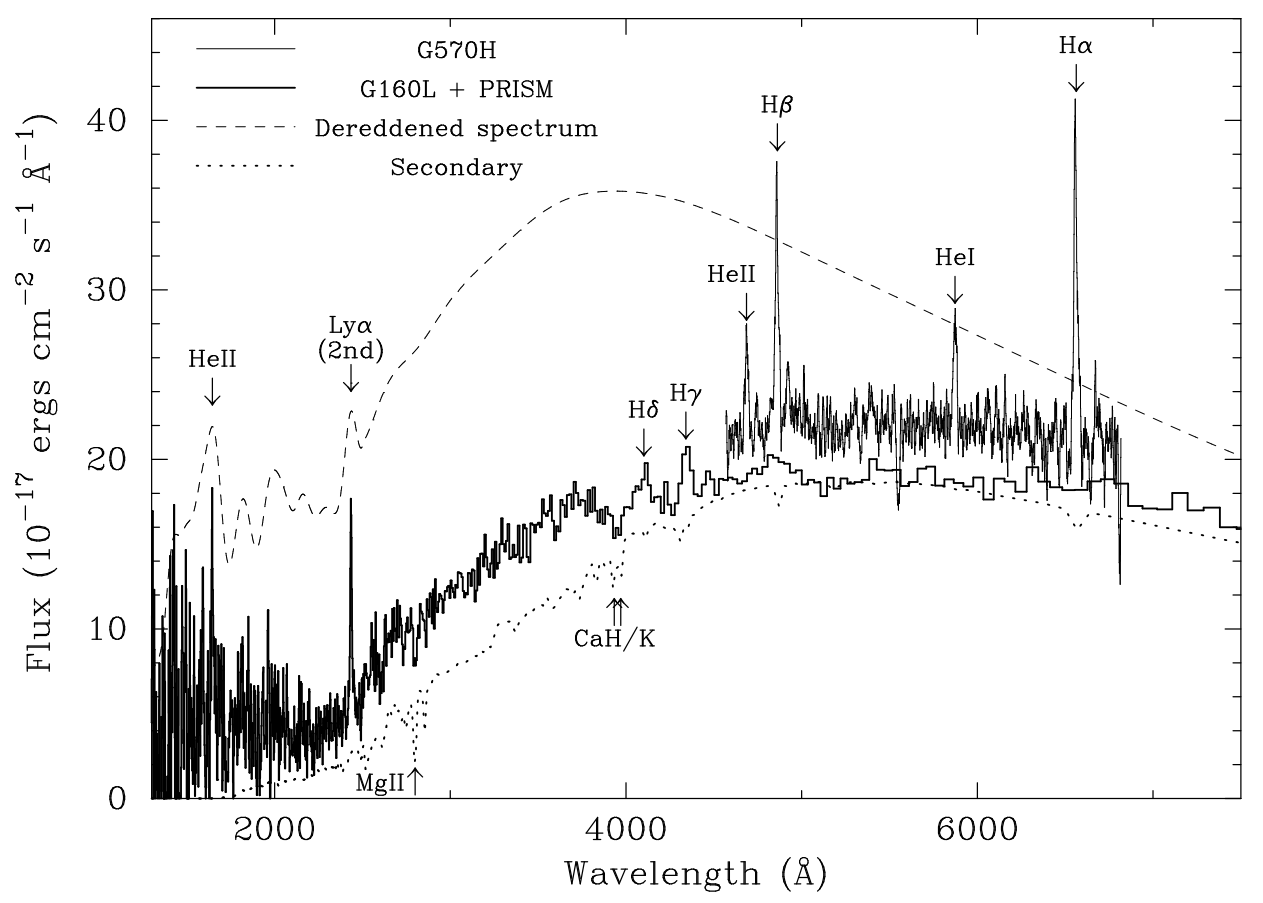

Fig. 1. - A plot of all the FOS data available for CV 1: the full G160L spectrum and the PRISM spectrum from $2510 \AA$ red-ward plus the red G570H spectrum from Cycle 4. Also shown is the dereddened smoothed continuum and the estimated Kurucz spectrum of the secondary (see text).

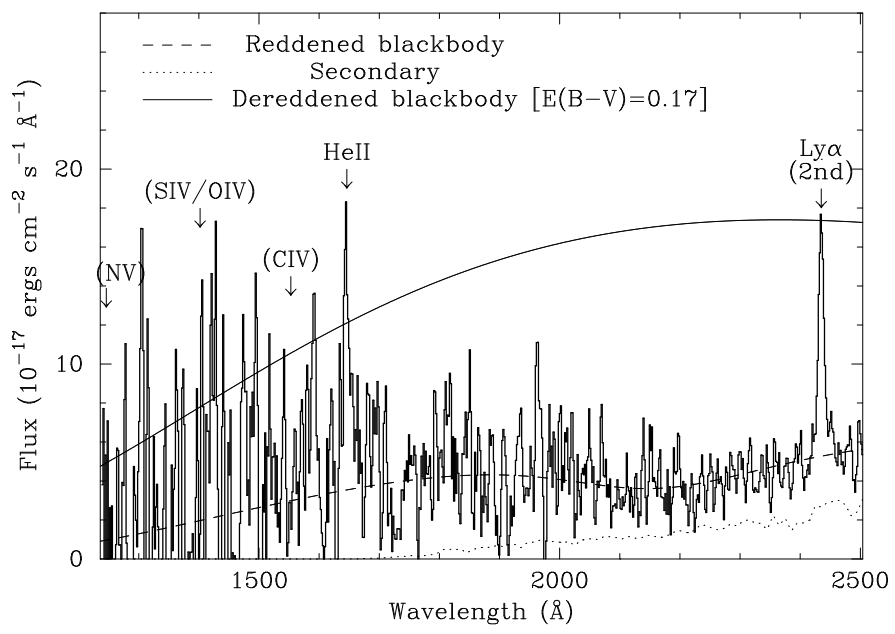

Fig. 2.- A close-up of the G160L spectrum. Also shown are a reddened blackbody fit to the UV spectrum (temperature $=12850 \mathrm{~K}$ ), dereddened spectra of this fit using three different values of $E(B-V)$ and the estimated Kurucz spectrum of the secondary. He II $\lambda 1640$ and Ly $\alpha$ in 2nd order are the only 2 emission lines detected. 


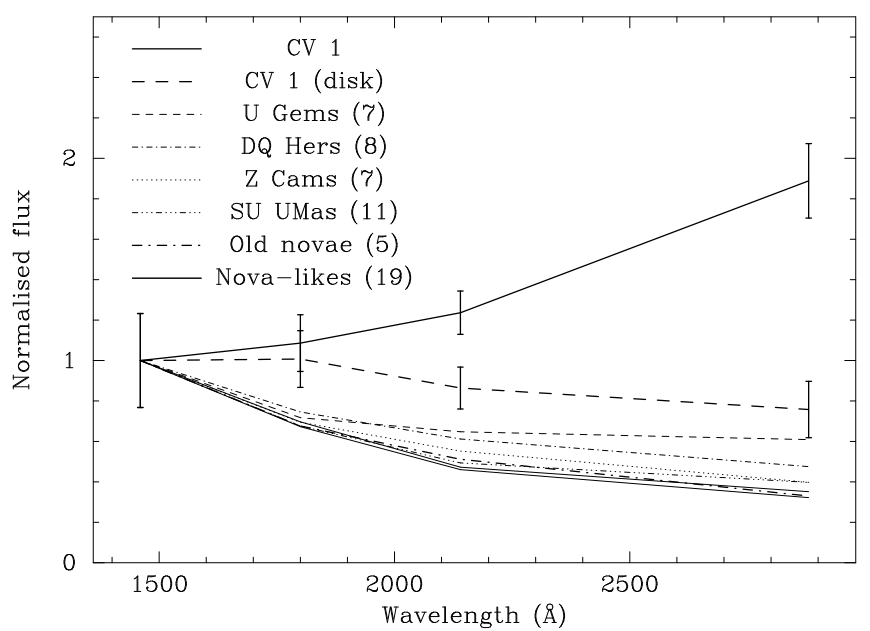

Fig. 3.- The dereddened UV fluxes for CV 1 and the CV 1 disk (total continuum minus the Kurucz spectrum of the secondary) along with fluxes for various CV classes (number of systems given in parentheses). The fluxes have been normalized by the flux at $1460 \AA$. The error bars combine uncertainties in the continuum level estimation, the reddening and the companion star estimate.

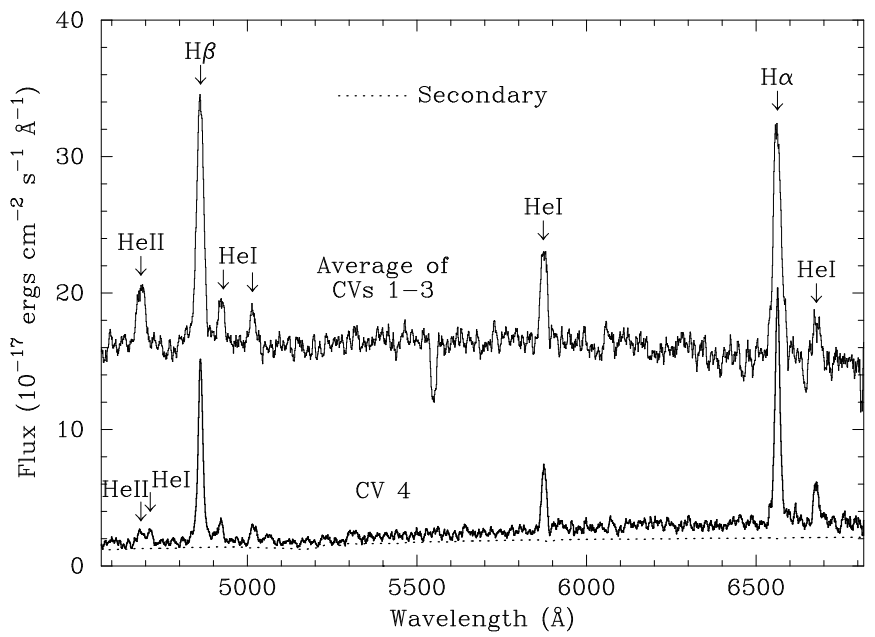

Fig. 4.- The diffuse light-corrected G570H spectrum of CV 4 and the average spectra of CVs 1-3 from GC95, where the continua of CVs 2 and 3 were normalized to that of CV 1 (the feature at $5550 \AA$ is an instrumental artifact). The dotted line shows the estimated contribution of the secondary for CV 4. The emission lines detected are labeled. Note the relative weakness of the He II $\lambda 4686$ line for CV 4 compared to CVs $1-3$, and the detection of HeI $\lambda 4713$. 


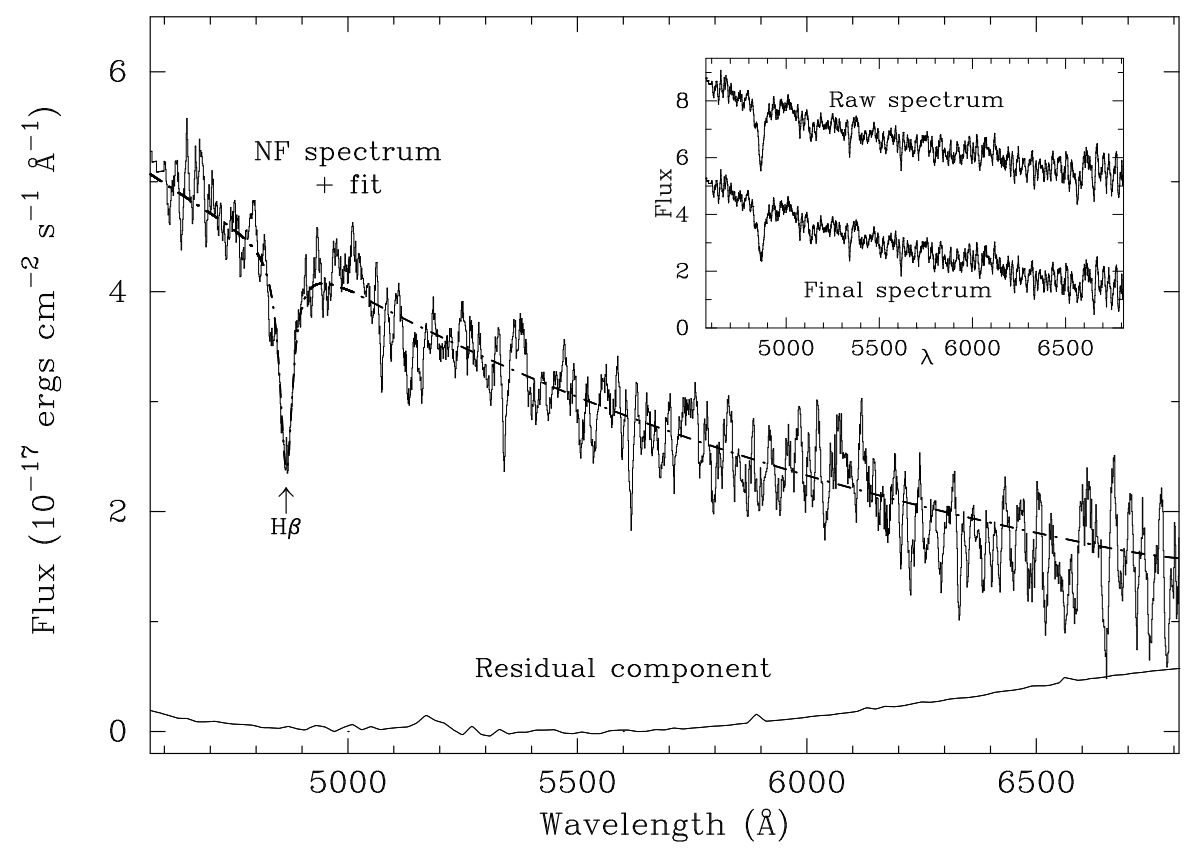

Fig. 5.- The G570H spectrum of the NF, after normalizing its continuum using the photometry of CG98. The model profile resulting from a fit to the $\mathrm{H} \beta$ line is also shown, plus the residual component described in the text. The inset shows a comparison between the raw spectrum and the normalized ("final") spectrum. Note the small difference in the $\mathrm{H} \beta$ line profile between the raw and final spectrum because of the removal of the spectral components from the neighboring star and diffuse light. 


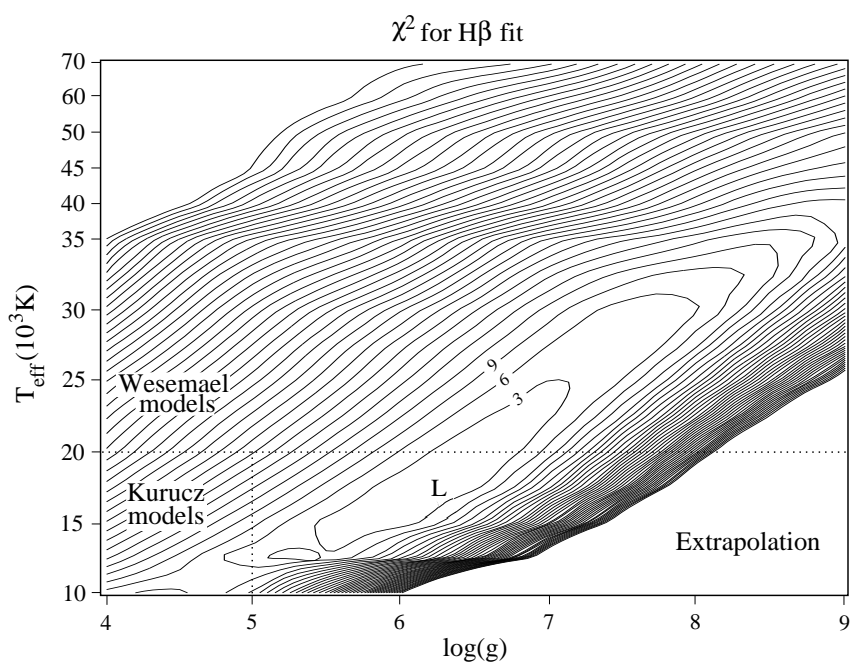

Fig. 6. - A contour plot of $\chi^{2}$ for the fit of the Wesemael and Kurucz line profiles to the $\mathrm{H} \beta$ line of the NF. The "L" shows the optimal solution and the dotted lines separate regions where the Wesemael and Kurucz models have been used, and where they have been extrapolated. The first $\left(\chi^{2}=3\right)$, second $\left(\chi^{2}=6\right)$ and fourth $\left(\chi^{2}=12\right)$ contour levels correspond roughly to $1 \sigma, 2 \sigma$ and $3 \sigma$ respectively.

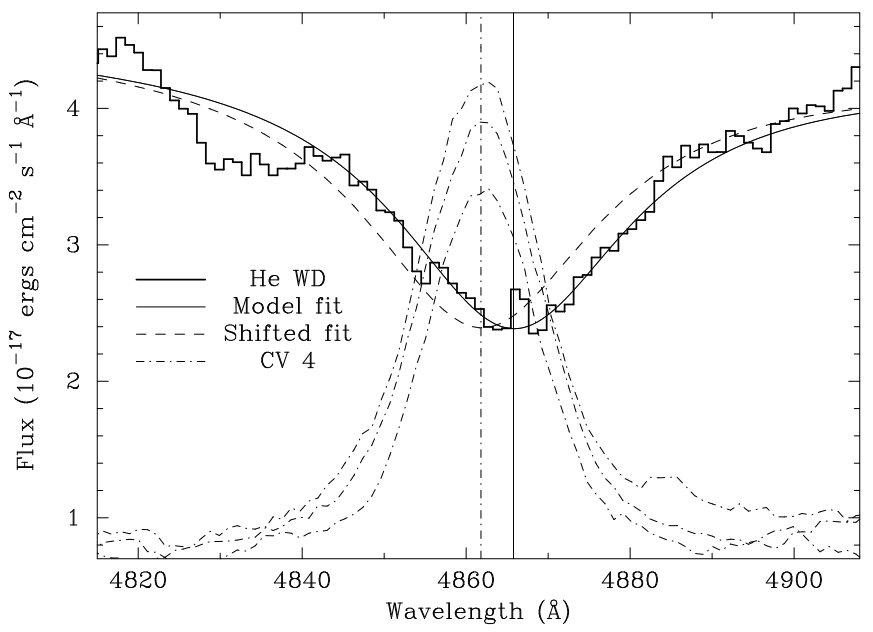

Fig. 7.- A closeup of the $\mathrm{H} \beta$ line profile of the NF. The noisy histogram plots the data, the smooth solid line is a Lorentzian model fit to the data, and the dot-dashed emission lines are from the three separate observations of CV 4 (our wavelength reference), normalized to fit in our figure. The dashed absorption line is the above Lorentzian fit but shifted to the wavelength of the CV 4 line, and the vertical lines show the central wavelengths of the NF and CV $4 \mathrm{H} \beta$ lines. 


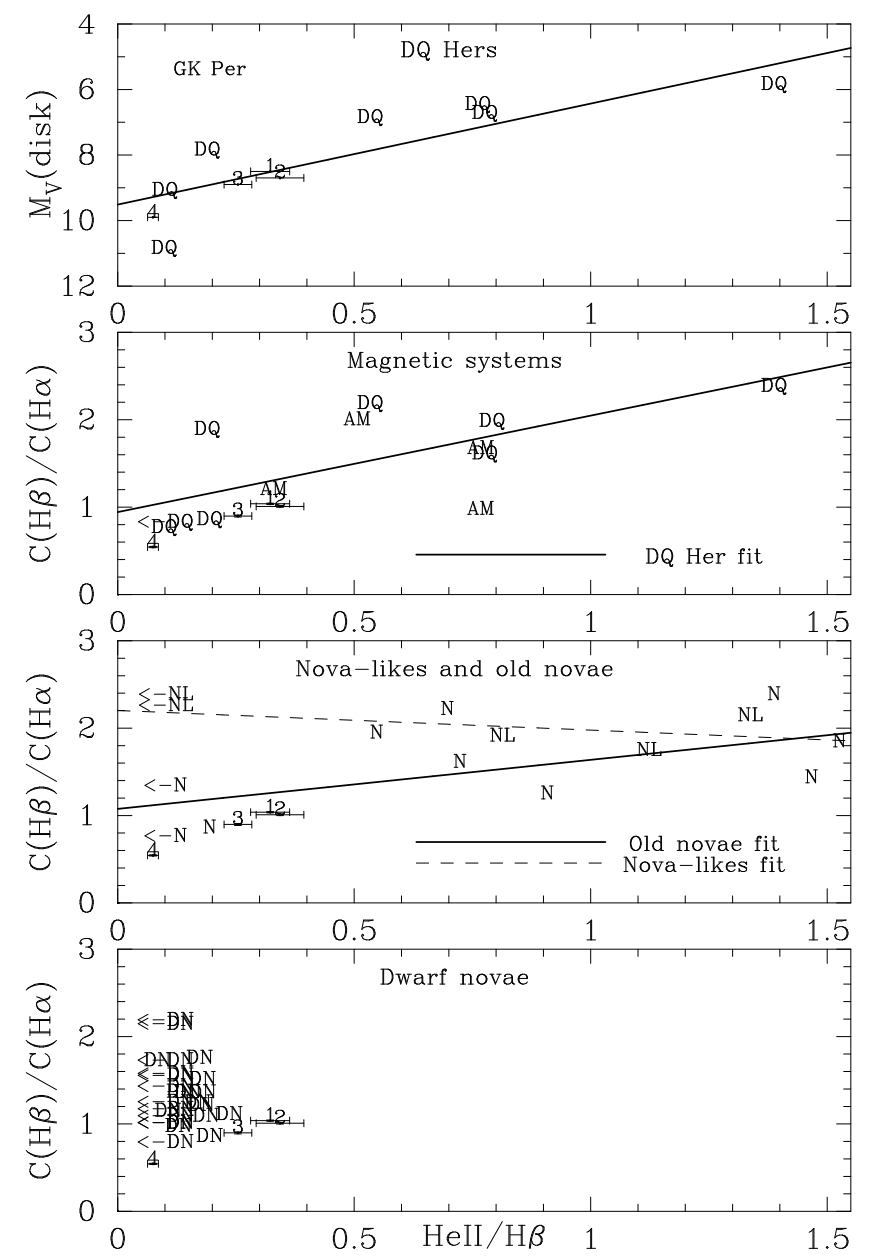

Fig. 8.- Plots of the continuum ratio between $\mathrm{H} \beta$ and $\mathrm{H} \alpha$ versus the $\mathrm{He} \mathrm{II} / \mathrm{H} \beta$ line ratio for CVs 1-4 along with several different classes of field CV (from Williams 1983). $\chi^{2}$ fitting straight lines are shown for individual CV classes where the linear correlation between the continuum and line ratios has absolute value $>0.5$. Systems with unmeasurable He II are plotted at He II/H $\beta=0.1$, and the $M_{V}$ (disk) values are taken from column 7 of Table 2 . 